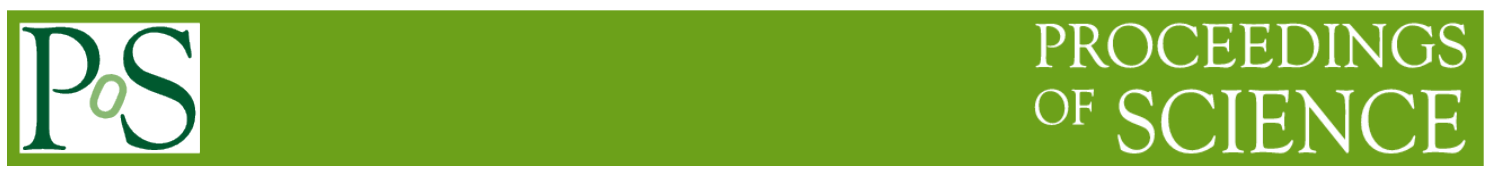

\title{
Study of solar transients causing GMSs with Dst $\leq-100$ nT during the period 1999-2010
}

\section{Rajiv Kumar ${ }^{1}$}

Rani Durgavati University Jabalpur

Madhya Pradesh,India

E-mail:captainrajiv@live.com

\section{N.K. Pandey}

Rani Durgavati University Jabalpur

Madhya Pradesh,India

E-mail:rajvij96@gmail.com

\section{Gajendra Singh}

Rani Durgavati University, Jabalpur

Madhya Pradesh, India

E-mail: pvcol legelgmail.com

The effect of solar features on geospheric conditions leading to geomagnetic storms(GMSS)with Dst index Dst $\leq-100 n T$ has been investigated using interplanetary magnetic field(IMF), solar wind data(SWP) and solar geophysical data with CMEs that erupted between 1999 and 2010, we considered all 51 events. The study investigated the relationship coronal mass ejection (CME) and their influence on Earth's geomagnetic field, i.e. storms and sub storms. The study is performed mainly considering intense geomagnetic storms that occurred during Solar Cycle 23 and ascending phase of 24 Solar Cycle. It has been analysed and estimated by cross correlation method that there is a delay of 17 to 96 hours in happening GMSs on the Earth after the happening of the CME on the sun.

Keywords: Coronal mass ejections; Solar Wind; Interplanetary Magnetic Field; Geomagnetic Storms.

35th International Cosmic Ray Conference - ICRC2017

10-20 July, 2017

Bexco, Busan, Korea

\section{${ }^{1}$ Rajiv Kumar}

(C) Copyright owned by the author(s) under the terms of the Creative Commons 


\section{INTRODUCTION}

The history of geomagnetism is more than 400 years old. Geomagnetic storms as we know them were discovered about 210 years ago. A severe geomagnetic storm may produce many harmful effects on earth, such as in Carrington event (1-2 September 1859), radiation hazards to humans, especially to astronauts, disruption occurred due to abnormal conditions in the interplanetary magnetic field (IMF) and solar wind plasma emissions caused due to the various communication, navigation and satellite control systems, damage of electric power grid as during 1-2 September 1859(the Carrington event) and so on [1]. Geo-magnetic storms generally originates from transient phenomena occurring on the surface of Sun[2]. The occurrence of prominences and flares is also associated with varying phases of sunspot cycle leading to the geomagnetic storms. The strength of interplanetary magnetic field (IMF) and its fluctuations have also shown to be most important parameter affecting the geomagnetic field condition. It is now a well established fact that the southward direction of interplanetary magnetic field, allows sufficient energy transfer from the solar wind into the Earth's magnetosphere through the magnetic reconnection process [3\& 4].

The study of these worldwide turbulence of Earth's magnetic field is important in understanding the mechanics of solar-terrestrial environment and furthermore because such storms can cause life threatening power outrage, satellite damage, communication failure and navigational problems $[2,3,4]$.Since the beginning of the space age, the cause of geomagnetic activity has been sought in a number of correlative studies [1].It is suggested that geomagnetic, activity is related to variety of interplanetary plasma/ field parameters, e.g. Solar wind velocity V, interplanetary magnetic field (IMF) $\mathrm{B}$ and $\mathrm{Bz}[\mathbf{1 , 2 , 4 ]}$.the strong geomagnetic disturbance is associated with passage of magnetic cloud [3,4], which causes intense and sever geomagnetic storms [5]. It is well established fact that solar wind is continuously emanating from the sun's outer corona and engulf the entire heliosphere. It mainly consists of hot electrons and protons flowing supersonically and caused due to extremely high coronal temperature helping ionized plasma to overcome the gravitation attraction of the Sun. The density and speed of this flow are highly variable and depend solely upon the conditions which have caused it to eject. The solar wind carries with it a huge amount of the magnetic field of the Sun, which when entering into the interplanetary medium is termed as Interplanetary Magnetic Field (IMF). The strength and orientation of this magnetic field associated with solar wind depend on its interaction between slow and fast solar wind originating from coronal holes and lead to creating co-rotating interaction region (CIR) [1].(SIDs). These are caused by the disturbances originated at solar atmosphere, interplanetary (IP) shocks and / or stream interfaces associated with high speed solar wind streams (HSSWS) [5,6] These are associated with Coronal holes $(\mathrm{CH})$, which occur in Polar regions or higher latitude. Fast CME produce transient IP shocks, which cause storm sudden commencement (SSC) at earth. Sometimes geo-magnetic storms are also associated with isolated disappearing filaments $[\mathbf{3 , 7 , 8 ]}$. The occurrence of prominences and flares is also associated with varying phases of sunspot cycle leading to the geomagnetic storms. The strength of IMF and its fluctuations have also shown to be most important parameter affecting the geomagnetic field condition. Southward direction of IMF allows sufficient energy transfer from the solar wind into the Earth magnetosphere through magnetic reconnection process []. As geo-magnetic storm lasts usually a few hours to several days in duration. However, sometimes the recovery phase of a geomagnetic storm lasts one to 


\section{Study of solar transients causing GMSs with Dst $\leq-100 n T$ during the}

period 1999-2010

Author(s)

two weeks or even for longer durations too. These kinds of long-duration events were termed as High-Intensity Long-Duration Continuous AE Activity events (HILDCAAevents). In a study of such events made by "Tsurutani and Gonzalez (1987)", it was suggested that continuous injections to the ring current take place during these events in such manner that the ring current does not, or cannot, decay rapidly $[9,10,11]$.Various studies have reported that these geo-effective events are further associated with CME's, solar flares, SEPs and also with other solar wind transients $[12,13]$. The influence of these solar ejectas not only significantly produces the depressions in the earth's ring current but modulates the cosmic ray intensity too. Its observed effects are Forbush decrease (Fd) event and Ground Level Enhancement (GLE) event $[\mathbf{1 5}, \mathbf{1 6}]$. The Forbush Decrease (Fd) is a transient decrease in the cosmic ray intensity which lasts for few hours to several days. On the other hand, the Ground Level Enhancement (GLE) event is a short -sharp enhancement in the cosmic ray intensity lasting for few minutes. GLE information is very useful not only for the understanding the Cosmic Ray Physics and particle modulation but to understand the solar - terrestrial environmental mechanism. Solar flares affect all layers of the solar atmosphere (photosphere, chromo sphere, and corona), when the medium plasma is heated to tens of millions of Kelvins and electrons, protons, and heavier ions are accelerated to near the speed of light. They produce radiation across the electromagnetic spectrum at all wavelengths, from radio waves to gamma rays, although most of the energy goes to frequencies outside the visual range and for this reason the majority of the flares are not visible to the naked eye and must be observed with special instruments. The frequency of occurrence of solar flares varies, from several per day when the Sun is particularly "active" to less than one every week when the Sun is "quiet", following the 11-year cycle (the solar cycle). Large flares are less frequent than maller ones. Solar activities vary with an 11-year cycle there are typically more sunspots on the sun, and hence more solar flares [17].

A coronal mass ejection (CME) is massive burst of solar wind, other light isotope plasma, and magnetic fields rising above the solar corona or being released into space. Recent scientific research has shown that the phenomenon of magnetic reconnection is responsible for CME and solar flares. When the ejection is directed towards the Earth and reaches it as an interplanetary CME (ICME), the shock wave of the traveling mass of Solar Energetic Particles causes a geomagnetic storm that may disrupt the Earth's magnetosphere, compressing it on the day side and extending the night-side magnetic tail. When the magnetosphere reconnects on the night-side, it releases power on the order of terawatt scale, which is directed back toward the Earth's upper atmosphere $[\mathbf{9 , 1 3}$. The relationship between solar flares and Coronal Mass Ejections (CMEs) is a big issue in solar physics (e.g., Gosling 1993; Hudson et al. 1995). Both of these phenomena often occur in conjunction but the relationship is not one to one; the exact nature of the flare and CME triggers and the relationship between the cause and consequence are still open and quite puzzling. "Kahler (1992)" pointed out that if the CME is associated with a flare then the CME originates in the explosive phase of the flare and such flares are long-decay events (LDEs); but the relationship of CMEswith impulsive flares is still unknown to us $[\mathbf{1 1}, \mathbf{1 8 , 1 9 ]}$. Solar flare and CME's are produced in sporadic solar eruptions. Solar flares and CME are in the larger ones. The distinction between solar flare and CME is that a solar flare is a sudden flash of electromagnetic radiation, whereas a $\mathrm{CME}$ is a mass motion in the solar corona that can be seen in a coronagraph. The spatial relationship between flare and CME depends on 


\section{Study of solar transients causing GMSs with Dst $\leq-100 n T$ during the}

period 1999-2010

the magnetic field configuration involved in the solar eruption process. Flares are perhaps originates from photosphere and CMEs from chromosphere [12]. The flares erupt from the intensely luminous area of the sun, whereas the CMEs are ejected from an bright and transparent layer of gas lying above and surrounding the photosphere. So flares can presumably trigger CMEs. However, there are also arguments that both phenomena might originate from the same active region of the solar disk even though they have different manifestation. Detailed explanations can be found in several studies [e.g., Kahler et al., 2001; Dorman, 2004; Yashiro et al., 2008; Belov, 2009]. Intense solar flares and fast CMEs consisting of entrained magnetic fields have enough potential to create turmoil in the earth's atmosphere [e.g., Manchester et al., 2005; Wang and Wang, 2006]. Namely, electromagnetic emissions produced by solar flares penetrate the Earth's atmosphere and change particle environment on the Earth, consequently disrupting radio transmissions. X-ray flares and CMEs may also cause sharp rise in cosmic ray intensities in the Earth. So, a study on characteristics of GLE- associate SEPs, X-ray flares, and CMEs can be useful for the understanding of cosmic rays and space weather.

The Dst index is taken as an indicator of geo-magnetically disturbed condition, as it represents the depressions in the ring current as a result of its interaction with the plasma signatures having their roots originated at solar surface or from some other exotic environment. We investigated various solar parameters/ interplanetary magnetic field components which were potentially geo- effective and occurred during the solar activity period of solar cycle-23and 24 .

In this chapter the statistical study has been performed to analyse these geomagnetic storms recorded by various geomagnetic observatories identified with the help of Disturbance storm time index (Dst).

\section{SELECTION CRITERIA AND DATA ANALYSIS.}

The part of irregular phenomena on the solar disc with the source that is responsible for SSC at the Earth. Variations of GMSs with SSNs have been investigated from 2000 to 2011. We used cross-correlation function because it shows correlations at different points of two waveforms thereby exhibiting all correlations between every two signals of the waveforms. The time-delay is then calculated as the time-length with respect to the specific wave point where the highest correlation is found. Crosscorrelation analysis provides correlations between data of two time series or waveforms. The observations of one data series are correlated with the observations of another data series at various delays and leads. Cross-correlations help identify variables which are leading indicators of other variables or how much one variable is predicted to change in relation with the other variable. The cross-correlation test of two time-series data sets involves many calculations of the coefficient ( $\mathrm{r}$ ) by time-shifting the one dataset relative to the other data set. Each shift is called a 'lag' and the lag time is simply the sampling period of the two time series data sets. A typical cross-correlation graph shows enough lags in both negative and positive directions to show the cyclical relationship of the two sets of data. Disturbances in the geomagnetic field are caused by fluctuation in the solar wind impinging on the earth. The disturbances We have analyzed the events represented by maximum. A list of magnetic storms, based on the Dst indices provided by the World Data Center for Geomagnetism, Kyoto, Japan through its World Wide Web ( and also from the Omni web data source maintained by National Geophysical Data Center (NGDC) : (http://www. ngdc. noaa. gov/ stp/SOLAR/ ftpsatenvir. html) is being compiled for this study for the period 2000-2011. As the study period refers to the 
interval solar cycle 23 and 24 . It is known that the intensity of solar parameters (for interplanetary magnetic field $\mathrm{B}, \mathrm{Bz}$ and solar wind velocity $\mathrm{V}$.) is registered by satellite at the geostationary orbit in the near Earth space whereas the magnetic field variation and ring current depressions are recorded by a network of observatories well located all over the world. This data is compiled by the world data center Kyoto, Japan and distributed to the world scientific community through its World Wide Web. Ultimately, there is a time-delay between the registration of Dst and the registration of solar parameters. To find out the time-delay a program is developed in $\mathrm{C}$ language. A list of magnetic storms, based on the Dst indices had been compiled for this study for the period 2000-2011. The Disturbance storm time index (Dst) is provided by the World Data Center for Geomagnetism at the University of Kyoto, Japan database (http://www.swdc.kugi. kyoto-u.ac.jp/ dstdir). Where the solar wind data have been compiled since 1963, using the observed data from 7 space satellites including the ACE, WIND and IMP. The Coronal Mass Ejection data used for the present study were adopted from the SOHO LASCO CME catalogue being maintained and provide by CD AW(http://cdaw.gsfc.nasa.gov/cme_list/halo/halo.html) .

\section{OBSERVATIONS}

The study of all 51 GMSs with Dst index depression $\geq(-) 100 \mathrm{nT}$ during the period 2000-2011 in $23^{\text {rd }}$ and $24^{\text {th }}$ Solar cycle has been investigated. To each of such GMSs, a CMEs could be responsible. The list of all such GMSs is compiled with its associated CME or CMEs because for severe storms it has been found more than one CME responsible.There is delay of time between commencement of CME and corresponding GMSs.This delay of time has been estimated by cross-correlation method. The Dst index is taken as the indicator of storm as registered by (http://swdcwww.kugi.kyotou.ac.jp) corresponding CMEs are identified by SOHO LASCO CATALOGUE and the (http://cdaw.gsfc. nasa.gov/CME_list) Fig .1 shows the comparative activity of the sun in $22^{\text {nd }}, 23^{\text {rd }} \& 24^{\text {th }}$ solar cycles .

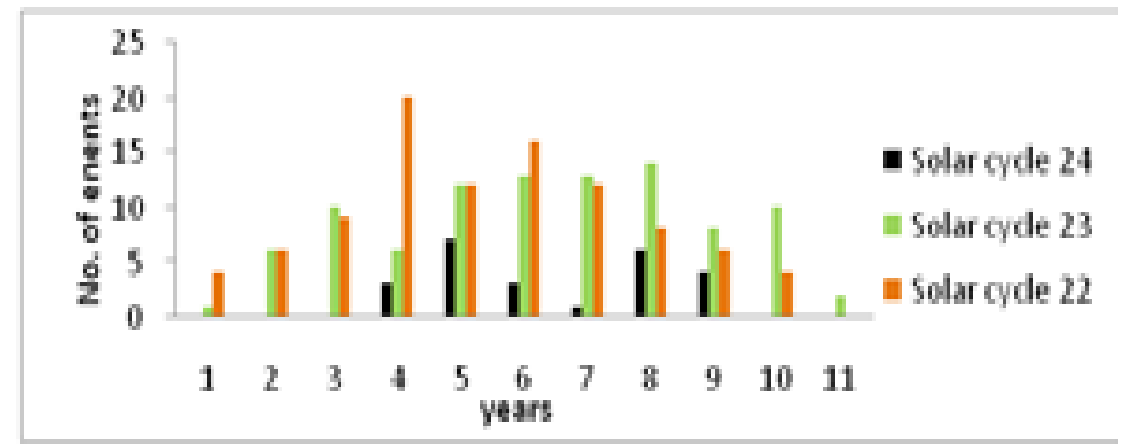

Fig .1 Comparitive activity of the Sun in 22-24 solar cycle

The sun is less active in $23^{\text {rd }}$ Solar cycle as compared to $22^{\text {nd. }} 24^{\text {th }}$ solar cycle is less acctive as compared to $23^{\text {rd }}$ solar cycle. In 25 th solar cycle the Sun would be even less active in $25^{\text {th }}$ Solar cycle and is considered as solar hybernation as predicted by Zharkova . Exactly below in fig 4.3 is the occurrence frequency which goes according to sunspot cycles in fig 4 Because of this Mini Ice age is predicted in the end of solar cycle 25 .

Electromagnetic fields and currents connect various regions of the Earth's near space environment extending up to the magnetopause. Realization of this fact has leaded to the concept of Global Electric Circuit (GEC) to describe the electromagnetic environment of the 
2:

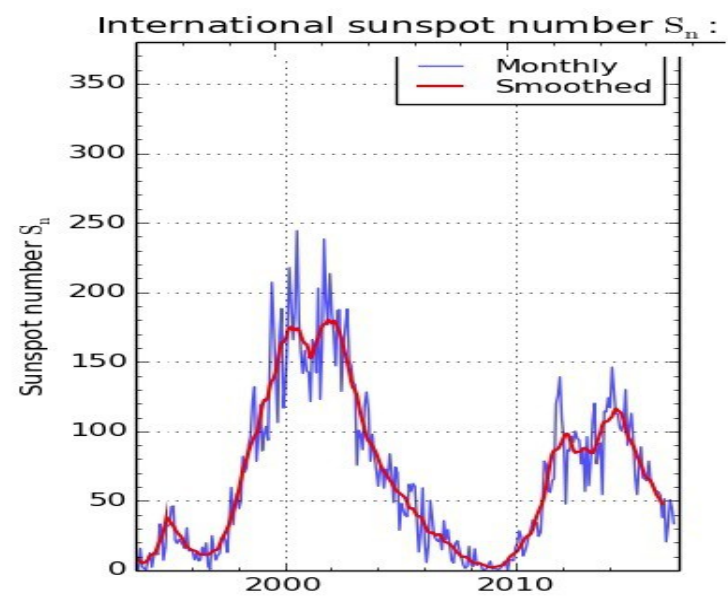

Fig

The sunspot cycle between 2000 and 2011

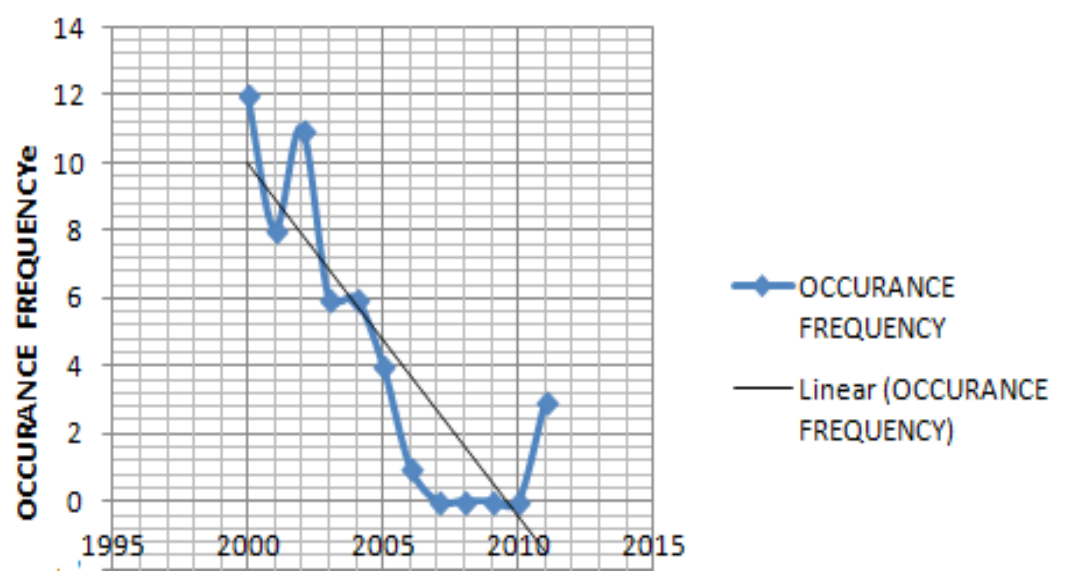

Fig 3: Occurrence frequency of GMSs during 2000to 2011.

from 01.09.200 to 31.10 .2002 is taken as the sample period in which there are high number of intense magnetic storms happened. The data is prepared for all $1456 \mathrm{hrs}$ duration

To handle such big data size we used a software www.wessa.net/rwasp_cross.wasp to calculate the cross correlation function. The result is as shown in Fig 4.4is the plot between the delay $\mathrm{d}$ and cross correlation function $\mathrm{r}(\mathrm{d})$. The plot shows four peaks one at about $17 \mathrm{hrs}$ other at $100 \mathrm{hrs}$ delay tird at about $140 \mathrm{hrs}$ and fift the most prominent at $170 \mathrm{hrs}$ delay time. This implies that the delay time is between 17 to $170 \mathrm{hrs}$ between the commencement of CME at sun and commencement of GMS at the Earth.

from 01.09.200 to 31.10.2002 is taken as the sample period in which there are high number of intense magnetic storms happened. The data is prepared for all $1456 \mathrm{hrs}$ duration

To handle such big data size we used a software www.wessa.net/rwasp_cross.wasp to calculate the cross correlation function. The result is as shown in Fig 4.4is the plot between the delay $d$ and cross correlation function $r(d)$. The plot shows four peaks one at about $17 \mathrm{hrs}$ other at $100 \mathrm{hrs}$ delay tird at about $140 \mathrm{hrs}$ and fift the most prominent at $170 \mathrm{hrs}$ delay time. This implies that the delay time is between 17 to $170 \mathrm{hrs}$ between 
Study of solar transients causing GMSs with Dst $\leq-100$ nT during the period 1999-2010

Author(s)

the commencement of CME at sun and commencement of GMS at the Earth.

Earth's atmosphere. Solar wind - magnetosphere - ionosphere. Magnetospheric substorms represent a global interaction between the solar wind, the magnetosphere, and the ionosphere. "Lakhina (1994)" reviewed the solar wind - magnetosphere- ionosphere coupling processes with emphasis on the nonlinear particle dynamics in the magnetotail [20]. Those aspects of the sub-storm processes which involve the chaotic dynamics are highlighted. Various methods based on nonlinear particle dynamics, linear prediction filtering techniques, phase space reconstruction techniques, and dynamical analogue models of geomagnetic activity are reviewed. It is shown that the solar wind magnetosphere - ionosphere system behaves as a strongly coupled nonlinear dynamical system which could be driven from regular to chaotic behavior with low dimensionality when the solar wind forcing is strong enough. The interplanetary parameters, e.g. interplanetary magnetic field, Btotal (nT) and its various components like $\mathrm{Bx}, \mathrm{By}, \mathrm{Bz}$ as well as solar wind parameters e.g. its velocity $(\mathrm{km} / \mathrm{s})$, ion density $(\mathrm{cc})$ and proton temperature $(0 \mathrm{~K})$ play a vital role in modulation process, the results are shown in Fig.4.5 The first panel of Fig. 4.6

Table 1

\begin{tabular}{|c|c|}
\hline Delay $(\mathrm{d})$ & $\begin{array}{c}\text { Cross correlation } \\
\text { factor } \mathrm{r}(\mathrm{d})\end{array}$ \\
\hline 0 & 0.030468073 \\
\hline 10 & 0.066864578 \\
\hline 20 & 0.070380774 \\
\hline 30 & 0.066894416 \\
\hline 40 & 0.036016085 \\
\hline 50 & -0.022675668 \\
\hline 60 & -0.0283989 \\
\hline 70 & -0.025507738 \\
\hline 80 & 0.034557041 \\
\hline 90 & -0.013987267 \\
\hline 100 & 0.068690875 \\
\hline 110 & 0.014296515 \\
\hline 120 & 0.021160296 \\
\hline 130 & 0.017586618 \\
\hline 140 & 0.108863415 \\
\hline 150 & 0.130325842 \\
\hline 160 & 0.281582659 \\
\hline 170 & 0.234953329 \\
\hline 180 & 0.040036109 \\
\hline
\end{tabular}

Fig 4 Plot of cross correlation coefficient r(d) verses Delay d 
Study of solar transients causing GMSs with Dst $\leq-100$ nT during the period 1999-2010

Author(s)
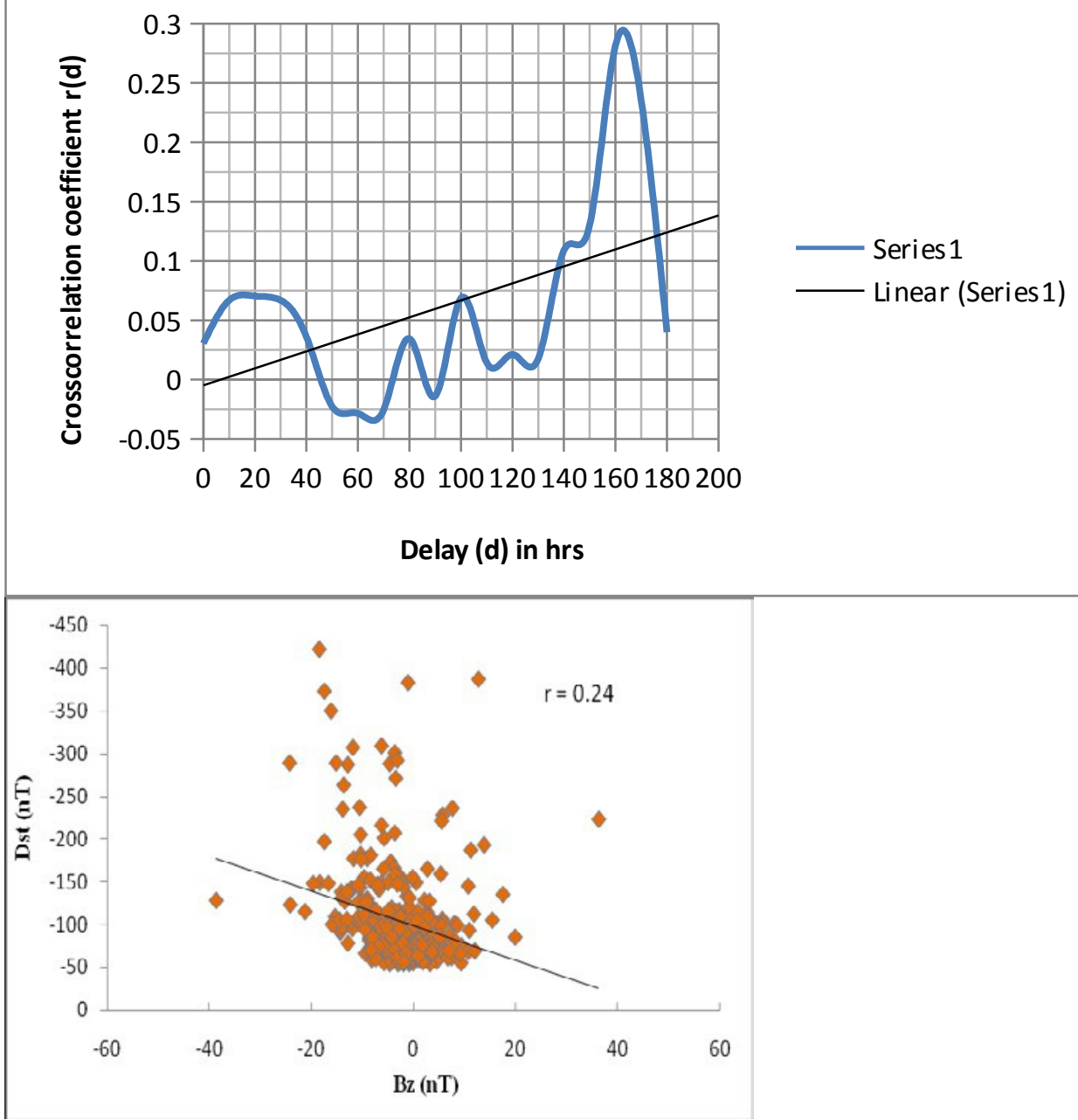

Fig 5 Correlation coefficient between $V$ and peak Dst

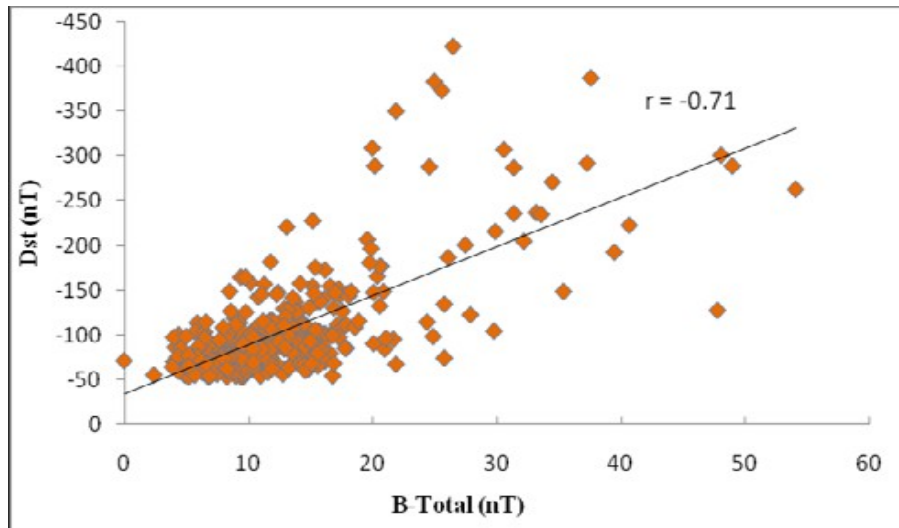

Fig 6 Wind speed $V(\mathrm{~km} / \mathrm{s})$ versus the maximum depressed negative Dst index measured in $\mathrm{nT}$ 


\section{Study of solar transients causing GMSs with Dst $\leq-100 n T$ during the}

period 1999-2010

Author(s)

depicts the maximum values reached by the solar wind speed $\mathrm{V}(\mathrm{km} / \mathrm{s})$ versus the maximum depressed negative Dst index measured in nT. The scatter is larger, with a wide range of velocities varying between 400 and $900 \mathrm{kms}-1$. The correlation coefficient between $\mathrm{V}$ and peak Dst has been found to be -0.36 while the correlation coefficient is -0.71 between the interplanetary magnetic field versus the maximum of negative Dst. Statistically. The correlation coefficient has been found to be reasonably high $(-0.71)$. This indicates the modulation effects within the Earth's geomagnetic field due to external parameters, like solar/interplanetary field.

\section{CONCLUSIONS}

From the rigorous analysis of data presented in the foregoing section, the following conclusions are drawn:

(i) It has been studied from the analysis of observed data that, $75 \%$ and $25 \%$ GMSs are occurred during maximum and minimum activity years of the $22^{\text {nd }}$ and $23^{\text {rd }}$ solar cycle respectively whereas, $73.2 \%$ and $26.8 \%$ GMSs have occurred during maximum and minimum activity years of the $24^{\text {th }}$ solar cycle. A peculiar result has been observed during the years 2000, 1994 when SSNs decrease very rapidly while the number of GMSs increase.

(ii) No distinct association between yearly occurrence of disturbed days and SSNs is observed. Some how, larger number of disturbed days have occurred during the years $2000,2001,2003$. Number of disturbed days vary with seasons. The distribution of disturbed days show a cyclic variation during the solar cycles.

(iii) It has been observed that for severe storms more than one CMEs are responsible

(iv) It has been also obseverd that Solar active is decreasing with solar cycles i.e. solar activity was more in $22^{\text {nd }}$ solar cycle than in $23^{\text {rd }}$ solar cycl, similarlythe solar acitivity is more in $23^{\text {rd }}$ solar cycle than in $24^{\text {th }}$ solar cycles. By this result it is predicted that solar activity would least in $25^{\text {th }}$ solar cycle, and Sun would be in the phase of Solar hibernation. Thus causing a mini ice age in $25^{\text {th }}$ Solar cycle.

(v) It has been analyzed and estimated there is a delay of 17 to 96 hours in happening of GMSs on the Earth after the happening of CME on the Sun.

(vi) It's analyzed that $63 \%$ GMSs are associated with halo or partial halo CMEs. However, few GMSs are not associated with any of the solar features on the visible side of the solar disc.

(vii) CMEs are related with eruptive prominence, solar flares, type IV radioburst and they occur at low helio-latitudes. It is observed that CMEs related GMS events are not always associated with HSSWSs.

(viii) In many individual events, the travel time between explosion on the Sun and maximum activity lies between 09 and $170 \mathrm{~h}$ causing GMSs at the Earth.

(ix) In the present study the correlation coefficient between $\mathrm{Bz}$ and Dst is found to be low (0.24). This that solar wind velocity and IMF southward magnetic field component $\mathrm{Bz}$ has no significant role in the initiation process of geomagnetic storm.

(x) In this study it is noticed that, Bz is not essentially peak at the time of Dst peak value. This shows time delay between $\mathrm{Bz}$ and Dst peak. It has been verified that geomagnetic storm intensity is correlated well with the total magnetic field of IMF better than southward component Bz of the IMF, density and solar wind velocity. 
Study of solar transients causing GMSs with Dst $\leq-100 n T$ during the period 1999-2010

Author(s)

\section{REFERENCES}

1. _I. Sabbah, Canadian Journal of Physics, vol. 78, pp. 293-302, 2000.

2. Hanaoka, Y and Shinkawa, 1998, Astrophys. J.,263.

3. C. Kuglin and D. Hines, "The phase correlation Image Alignment Method", Proc. Intl. Conf. Cybernetics and Socity, pp.183-165, 1975.

4. Canfeild, R.V. Hudson, H.S. McKenzie, D.E. 1999, Geophys. Res. ett., 26, 627.

5. I. G. Richardson and H. V. Cane, " Geoeffectiveness (Dst and Kp) of interplanetary coronal mass ejections during 1995 - 2009 and implications for storm forecasting", Space Weather, 9, S07005, Doi: 10.1029/2011 SW 000670 (http://dx.doi.org/ 10.1029/2011 SW000670). 2011.

6. Green, L.<. Lopez Fuentes, M.V. Mandrini, CH Demoulin, P., Van Driel-Gesztelyi, L. Culharo, J.L. 2002 Solar Phys. 208,

7. Devore, CR. 2000, Astrophys, J. 539, 944.

8. Gopalswamy, N., and Thompson, B.J., 2000, JASTP, 62,1457.

9. Gopalswamy, N., 1999, in NRO Report No. 479,p141.

10. Gopalswamy, N., Yashiro, S., Kaiser, M.L. and Bougeret, J - L, 2001, J. Geophys. Res., 106, 29261.

11. Gopalswamy, N. Yashiro, S. Liu. Y., Michalek, G., Vourlidas, A., Kaise, M.L. and Howard R.A. 2005 J Geophys. Res., (in press)

12. Demoulin, P., Mandrini, C.H. Van Driel-Gesztelyi, L., et al., 2002, Astron Astrophys., 382, 650 .

13. Gray G. A. 2001, Solar Phys. 203,71

14. Hanaoka, Y., et al., 1994 PASJ, 46,205

10 
Study of solar transients causing GMSs with Dst $\leq-100$ nT during the period 1999-2010 Author(s)

15. A. V. Belov, "Properties of solar X-ray flares and proton event forecasting", $A d$ vances in Space Research, vol. 43, pp. 467 - 473, 2009.

16. Gold, T., 1958 (ed by Lehnort. B) Cambridge Unipress, Cambridge, 275.

17. Gopalswamy, N., et al. 1996, New Astron., 1207.

18. A. S. Goshtasby, S. H. Gage, and J. F. Bartholic, IEEE Trans. Pattern Analysis and Machine Intelligence, vol. 6, iss. 3, pp. 374-378,1984.

19. Howard R.A. Michels, D.J. Sheeley, Jr. and Koomen, M.J. 1982 Astrophys. J. 263, L101..

1. 